\title{
Morbidity and Mortality of Rheumatic Valvulopathy Surgery in Young Adults in Senegal
}

\author{
Ciss Amadou Gabriel ${ }^{1, *}$, Diop Momar Sokhna ${ }^{1}$, Sow Ndeye Fatou ${ }^{1}$, Ba Pape Salmane ${ }^{1}$, \\ Diagne Papa Amath', Diatta Souleymane', Gaye Magaye ${ }^{1}$, Leye Mohamed ${ }^{3}$, Sene Etienne Birame ${ }^{2}$, \\ Dieng Papa Adama ${ }^{1}$, Ndiaye Assane ${ }^{1}$, Ndiaye Mouhamadou ${ }^{1}$ \\ ${ }^{1}$ Department of Cardiovascular and Thoracic Surgery, Dakar University, Dakar, Senegal \\ ${ }^{2}$ Department of Intensive care and Anesthesiology, Dakar University, Dakar, Senegal \\ ${ }^{3}$ Department of Cardiology, Dakar University, Dakar, Senegal
}

Email address:

cissgaby@yahoo.fr (C. A. Gabriel), momarsokhna08@yahoo.fr (D. M. Sokhna), zenefes@gmail.com (S. N. Fatou), mansalb@yahoo.fr (Ba P. Salmane), diagnepapaamathdiagne@gmail.com (D. Papa Amath), soul.diatta@yahoo.fr (D. Souleymane), mgaye99@yahoo.fr (G. Magaye), leyemouhamed@gmail.com (L. Mohamed), biramsene@hotmail.com (S. E. Birame), padiengsala@yahoo.fr (D. P. Adama), lazanat@yahoo.fr (N. Assane), mondiaye@orange.sn (N. Mouhamadou)

${ }^{*}$ Corresponding author

\section{To cite this article:}

Ciss Amadou Gabriel, Diop Momar Sokhna, Sow Ndeye Fatou, Ba Pape Salmane, Diagne Papa Amath, Diatta Souleymane, Gaye Magaye, Leye Mohamed, Sene Etienne Birame, Dieng Papa Adama, Ndiaye Assane, Ndiaye Mouhamadou. Morbidity and Mortality of Rheumatic Valvulopathy Surgery in Young Adults in Senegal. International Journal of Cardiovascular and Thoracic Surgery.

Vol. 4, No. 1, 2018, pp. 10-13. doi: 10.11648/j.ijcts.20180401.13

Received: December 21, 2017; Accepted: January 16, 2018; Published: February 8, 2018

\begin{abstract}
Rheumatic heart valve disease is the most important cardiovascular diseases in young African people. It's a cause of a heavy morbidity and mortality. The aim of this study is to evaluate the morbidity and mortality of valve surgery in young adults. It was a retrospective and monocentric study over 2 years. We had evaluated 69 patients with an average age of 32 years [16-63years]. Epidemiological, clinical, paraclinical, interventional and post interventional data were collected from archived patient records. The processing of this data was treated by SPSS v 16 and Excel software. Stage III dyspnea was the main symptom. Risk factors for postoperative mortality were cardiac insufficiency $(\mathrm{p}=0.006)$ and aortic valve position $(\mathrm{p}=0.009)$. Postoperative complications were dominated by hemodynamic instability $(23 \%)$, pulmonary complications $(20 \%)$ and infectious complications (3\%). Postoperative mortality was $5.8 \%$. Our late mortality was $2.9 \%$. Two cases of minor accidents to anticoagulation drugs were noted. The evolution was favorable for $85.5 \%$ of patients. In Africa, rheumatic heart disease remains the most common valvular heart disease with high morbidity and mortality. Cardiac surgery when it is available improves the quality of life of patients. The high cost of this surgery makes it's not always available. The prophylaxis of streptococcal bacterial attacks remains the best way for prevention.
\end{abstract}

Keywords: Morbidity, Mortality, Cardiac Surgery, Rheumatism Heart Disease

\section{Introduction}

Cardiovascular diseases are the main causes of mortality in the world. In the western countries coronary lesions are the most prevalent. In sub-Saharan Africa, rheumatic heart valve disease remains a major public health problem [1]. In Africa cardiac heart surgery is recent. In Senegal, the first open heart surgery was done in 1996. In sub-Saharan Africa they are few centers wish practice routinely cardiac surgery. Valve lesions are severe there are due to the recurrent rheumatic fever attack [3]. In the absence of surgical treatment, the morbidity and mortality of rheumatic heart disease remains high [1].

The aim of this study was to evaluate the morbidity and mortality of cardiac valve surgery in this pathology. 


\section{Method}

This is a retrospective single-center study over 2 years (January 2014 - December 2015). It included all patients over the age of 16 who had undergone valvular heart surgery at Dakar's thoracic and cardiovascular surgery department. A total of 69 patients were included in the study (42 women and $27 \mathrm{men})$. The average age was 32 years old [16 - 63 years old]. The etiology was rheumatic in all patients. Dyspnea was the main functional symptom. She was present in all patients. It ranged from stage II (34.7\%) to IV (1.4\%) NYHA. Previous cardiac decompensation was found in $42 \%$ of cases. All patients were stable with diuretic therapy and $94 \%$ of them had progressed to stage II NHYA. A history of recurrent angina was found in $55 \%$ of patients and arthralgia was found in $43.5 \%$ of patients. Two patients had been treated and cured for infectious endocarditis. Two other patients had previous cardiac surgery ( 1 case of mitral plasty and 1 case of mitral valve replacement). The ECG showed left atrial hypertrophy in $65 \%$ of patients. Atrial fibrillation was present in $45 \%$ of cases. The diagnosis of valvulopathy was confirmed by transthoracic ultrasound ETT). Mitral disease was found in $94.2 \%$ of patients. Aortic valve disease was confirmed in 15 patients $(21.7 \%)$. Significant tricuspid insufficiency was found in 25 patients $(36.2 \%)$. The degree of severity was variable. She was always associated with mitral and / or aortic disease. Hypertrophy of left atrium was found in $84 \%$ of cases, with a maximum diameter of $91 \mathrm{~mm}$ and an average diameter of $56 \mathrm{~mm}$. Left ventricular function (LVEF) was moderately impaired in $8.7 \%$ of patients. Ten patients (14.5\%) had impaired right ventricular function. We found severe pulmonary arterial hypertension (PAH) in 55\% of patients. It was isosystemic in four patients. Mean systolic pulmonary arterial pressure (PAPS) was estimated at 60 $\mathrm{mmHg}(23-127 \mathrm{mmHg})$. Sequelae of infectious endocarditis were found in $7 \%$ of patients.

All patients underwent extracorporeal circulation (ECC). The approach was via a vertical median sternotomy. The mitral valve was approached by the left atrium and the aortic valve by a transverse aortotomy. The myocardial protection was made by anterograde cold crystalloid cardioplegia associated with local protection by the Schumway method. Moderate hypothermia completed this myocardial protection. The mean aortic cross clamping time was 71 minutes [33-214 $\mathrm{mm}]$. The mean extracorporeal circulation (ECC) time was 95 minutes [50 - $343 \mathrm{~mm}$ ]. An incident per ECC was noted, as a defusing of the pump by disconnection of the venous line. The intervention focused on the mitral valve alone in 30 patients (43.4\%). This mitral surgery consisted of valve replacement in 26 patients and mitral plasty in 6 patients. Mitral valve replacement with tricuspid De Vega plasty was performed in 24 patients $(34.7 \%)$. the mitral valve was replaced by a mechanical prosthesis in all cases. A mitral plasty was performed in $8.7 \%$ of cases (6 patients). It was an annulopasty with homemade ring. In some cases we make an enlargement of the posterior valve with pericardium, transfer or section of secondariescordage, and cleft closure. The intervention focused on the aortic valve alone in 4 patients (5.8\%). Aortic and mitral valve replacement was performed in 10 patients $(14.5 \%)$. This dual aortic and mitral valve replacement was associated with tricuspid plasty in 1 patient (1.4\%). The aortic valve was replaced by a mechanical prosthesis in all cases.

The statistical analysis of the data had used the Epi Info7 software, SPSS, Stata, and the Excel spreadsheet. Under Epi Info, the Data Analysis tab, which has several features, was used to tabulate descriptive statistics and intersections between variables. The data obtained were compared with those obtained by SPSS and Stata software. The P-values of the different crosses were obtained through the Stata software. These values are compared with those of SPSS. The Chi-square test, with the Pearson coefficient as a coefficient, was used for the determination of P-values.

\section{Results}

Our operative mortality involved two patients $(2.9 \%)$. Both showed multiple pericardial adhesions. During adhesiolysis, one had a ventricular fibrillation followed by cardiac arrest that could not be recovered. The second patient had a drop in blood pressure and ventricular tachycardia rhythm disorders during pericardial detachment. After surgery, he had very severe left ventricular dysfunction. Extracorporeal circulation support was continued two hours after surgical procedure without improvement of the left ventricular function. The death occurred when the ECC was stopped.

The postoperative mortality involved four patients (5.8\%). Two patients had a pulmonary hypertension attack with right ventricular dysfunction. Two others had hemodynamic instability refractory to catecholamines. Postoperative morbidity was dominated by haemodynamic complications (23.2\%), followed by respiratory $(20.2 \%)$ and infectious (2.9\%) complications. These different complications were listed in Figure 1.

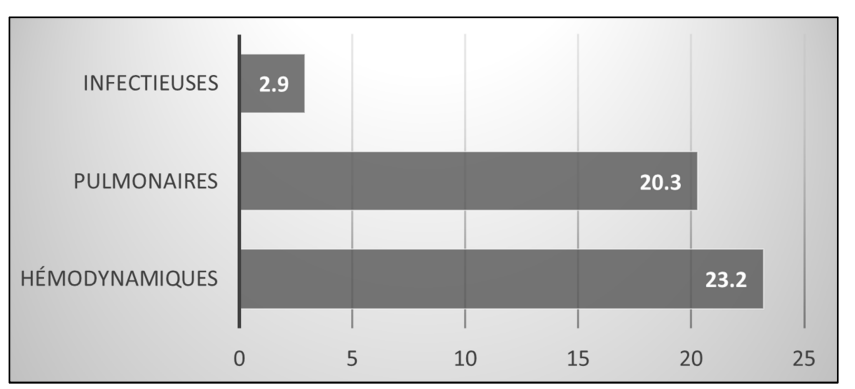

Figure 1. Distribution according to the type of postoperative complications.

We observed $2.9 \%$ superficial infection of the surgical site. No germ was indentified. The evolution was favorable under antibiotics and regular local care. Two cases of minor accidents with vitamin $\mathrm{K}$ antagonists were noted; they occurred in the second and fifth months postoperatively. The evolution was favorable for $85.5 \%$ of patients. Living patients had no recurrence of dyspnea. An apexal systolic 
murmur reappeared in five patients $(7.2 \%)$, indicating a tricuspid leak, which was not associated with signs of cardiac congestion. An electrical anomaly was found in 28 patients (40.6\%). It was a pre-existing ACFA in 23 patients $(33.3 \%)$ and appeared post-operatively in one patient $(1.4 \%)$. A patient undergoing mitral valve and tricuspid repair had a type $3 \mathrm{AV}$ block and had been paired with a dual chamber pacemaker.
The mean duration of follow-up was 8 months [1-22 months]. Two patients $(2.9 \%)$ died during follow-up. One patient died in the second postoperative month as a result of severe malaria. The second patient died at the 10th postoperative month, of an infective endocarditis on bioprosthesis. A patient was lost to follow-up after the 3rd month postoperatively. Actuarial survival is illustrated in Figure 2.

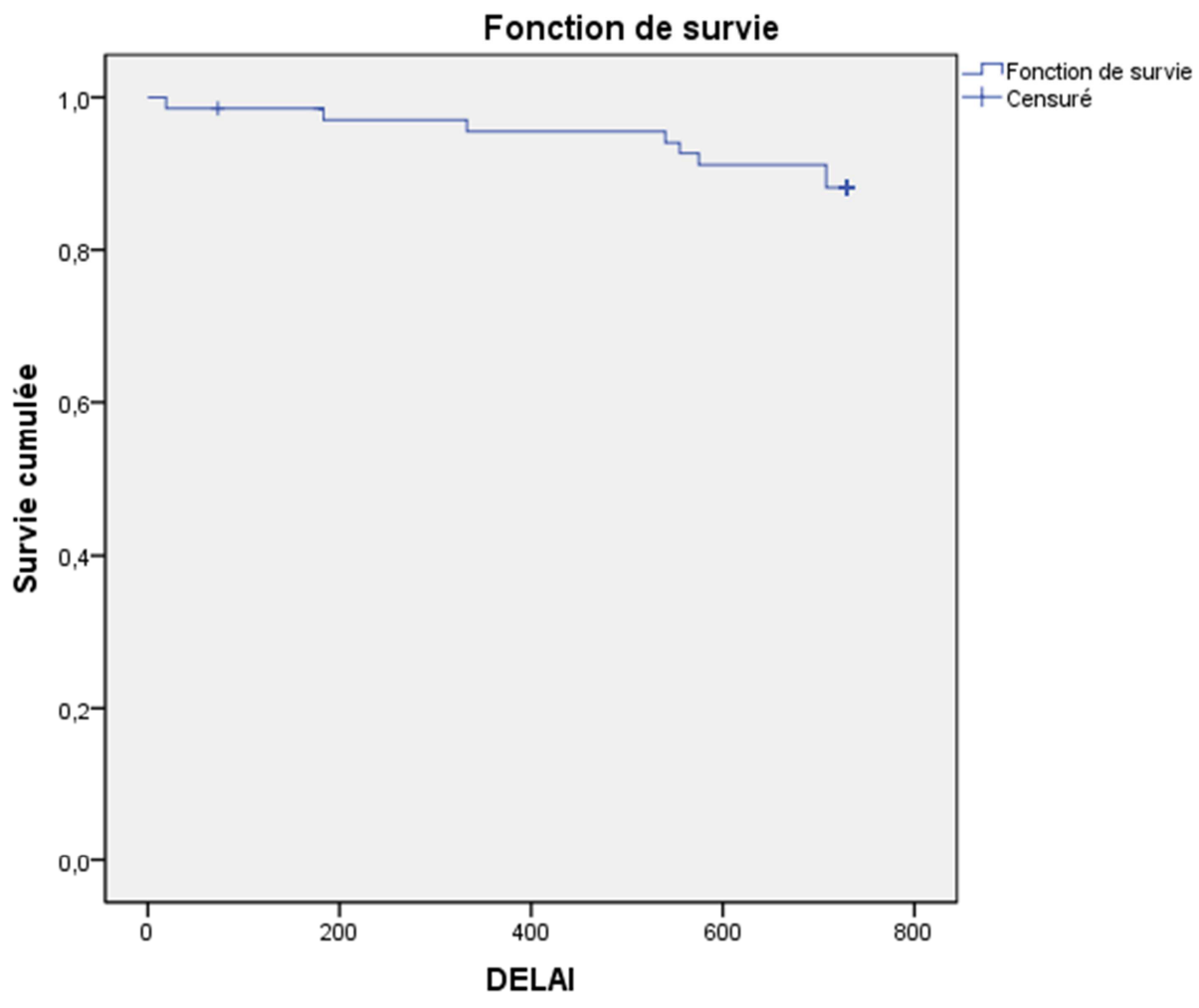

Figure 2. Kaplan Meir Survival Curve.

\section{Discussion}

Valvulopathy play an important role among cardiovascular diseases in Africa. Of the estimated 20 million rheumatic heart diseases in the world and the 500,000 deaths attributed to them, $95 \%$ occur in underdeveloped or developing countries [1]. Sub-Saharan Africa is concerned by half of the cases in the continent [1]. Unlike developed countries where degenerative causes are predominant, in sub-Saharan Africa infectious diseases are more common [2, 3]. Thus valvulopathies occur in a young population with a low socioeconomic level. The diagnosis of valvulopathy is discussed on clinical examination and confirmed by TTE. At the end of these evaluations, the therapeutic indications are codified by guidelines with class A evidence levels. Several factors of poor prognosis have been listed in our series. Cardiac decompensation would not increase the risk of hemodynamic complications $(\mathrm{p}=0.081)$, but would affect the death rate $(\mathrm{p}=0.006)$. ACFA remains the most common rhythm disorder. This frequency is also demonstrated by a number of series [4, 5]. This complete arythmia by atrial fibrillation is often associated with mitral attacks. It significantly alters the quality of life of the valvular patient [4]. We do not find a statistical correlation between preoperative ACFA and hemodynamic complications or postoperative mortality. Severe $\mathrm{PAH}$ was common in our series. Severe PAH greatly increases the operative risk $[6,7]$. We did not observe a statistical correlation between PAH and postoperative complications. Valve replacement is the most common procedure. We have a risk of hemodynamic complications related to a mitral position of the prosthesis ( $p$ $=0.0001)$ or to aortic position of the prosthesis $(\mathrm{p}=0.001)$ and a risk of death related to the aortic position of the prosthesis $(p=0.009)$. Mitral plasties were rare in our series $(8.7 \%)$. The literature describes poorer results when the plasty is performed on a rheumatic valve in adults [8]. Significant retractile fibrosis of the valvular system and valvular calcification don't allowany repair. Concerning the tricuspid valve, we practice the plasty technique according to De Vega technique. It is accessible and gives good results with a low economic cost, however the long-term results are disappointing unlike the use of ring. Our postoperative complications were hemodynamic, pulmonary and infectious. Hemodynamic complications in cardiac surgery are largely induced by CPB. They are also due to anesthesia and surgery 
itself [7]. The use of inotropes is of great help [7, 10]. Pulmonary complications, with an incidence of 8 to $10 \%$, are the second most common source of postoperative morbidity after cardiac complications [10]. Pleural effusion predominated in our series with a rate $(11.6 \%)$ much lower than reported in the literature (40-50\%) [10]. They occurred most often on the right contrary to the data of the literature [10]. Operative and postoperative mortality was in the meantime similar series of studies. Mortality reported in the series varies considerably by geographic location. In underdeveloped countries, rates between 1 and $7.8 \%$ are noted $[11,12,13]$. Death in one of the patients occurred during the semi-emergency intervention after non-structural prosthetic dysfunction. The operative mortality of repeat surgery in Africa is $13.8 \%$ and the emergency response rate is $24 \%$ [14]. We had two late deaths. One of the patients died from infective endocarditis on a mitral bioprosthesis. The overall incidence of prosthetic valve endocarditis varies between 1 and $4 \%[15,16]$. Their mortality rate is high, higher than $50 \%$ [15]. The second patient died from severe malaria.

\section{Conclusion}

In Africa, rheumatic heart disease remains the most common valvular heart disease with high morbidity and mortality. Cardiac surgery when it is available improves the quality of life of patients. The high cost of this surgery makes it's not always available. The prophylaxis of streptococcal bacterial attacks remains the best way for prevention.

\section{References}

[1] Monsuez. J. Valvulopathies Rhumatismales. AMC Pratique, 2011; 200 (4): 28-31.

[2] Roudaut. R., Dijos. M., Arsac. F., Reant. P., Lafitte S. Pathologie valvulaire: 50 ans de progrès AMC pratique, 2011; 200 (3): $21-23$

[3] Essop MR., Nkomo V. T. Rheumatic and NonrheumaticValvular Heart Disease Epidemiology, Management, and Prevention in Africa. Circulation, 2005; 112 (9): 3584-91.

[4] Lee J. W., Park NH; Shoo SJ; Jo MS; Song H; Song MG. Surgical Outcome of the Maze Procedure for Atrial
Fibrillation in Mitral Valve Disease: Rheumatic Versus Degenerative. The Annals of Thoracic Surgery, 2003; 75 (5): $57-6$.

[5] Mbaye A.; Pessinaba S; Bodian M; Ndiaye MB; Mbaye F; Kane A; Diao M. La fibrillation atriale, fréquence; facteurs étiologiques, évolution et traitement dans un service de cardiologie de Dakar, Sénégal. Pan African Medical Journal, 2010; 6, 16 (11): 1-11.

[6] Antunes M. J. Pulmonary Hypertension in Valve Disease: A beast of the past? The Journal of Thoracic and Cardiovascular Surgery, 2016; 151 (2): 1300-1.

[7] Cannesson M., Bastien O., Lehot J. J. Particularités de la prise en charge hémodynamique après chirurgie cardiaque. Réanimation, 2005; 14 (9): 216-24.

[8] Aubert S., Praschker B. L., Coignard E., Gandjbakhch I. La plastie mitrale dans tous ses états. MT cardio, 2008; 4 (6): 291-296.

[9] Antunes M. J. DeVegaAnnuloplasty of the Tricuspid Valve: Operative Techniques. Thoracic and Cardiovascular Surgery, 2003; 8,4 (8): 169-76.

[10] Chassot P. G., Bovi M., Mustaki J. P. Complications après chirurgie cardiaque. Précis d'anesthésie cardiaque, 2012; 23 (65): 1-65.

[11] Samiei N; Hakimi MR; Peighambari MM; Alizadeh-Ghavidel A; Hossein S. Surgical outcomes of heart valves replacement: A study of tertiary specialied cardiac center. ARYA Atheroscler, 2014; 10 (5): 233-7.

[12] Carapetis J. R; Powers JR; Currie BJ; Sangster JF; Begg A; Fisher DA; Kilburn CJ; Borrow JNC. Outcomes of Cardiac Valve Replacement for Rheumatic Heart Disease in Aboriginal Australians. Asia Pacific Heart J, 1999; 8, 3 (10): 138-47.

[13] Alizzi A. M., Knight J. L., Tully P. J. Surgical challenges in rheumatic heart disease in the Australian indigenous population. Heart, Lung and Circulation, 2010; 19 (4): 295-8.

[14] Kangah M. K; Souaga AK; Amani KA; Kiroua-Kamenan YA; Katche KE; Meneas GC; Kendja KF. La chirurgie valvulaire itérative en Afrique: indications, résultats et facteurs de risque. Chirurgie Thoracique Cardio-Vasculaire, 2010; 15 (4): 93-6.

[15] Chapelon C., Raguin G., Ziza J. M., Piette J. C., Godeau P. Endocardites infectieuses sur prothèses valvulaires. La revue de médecine interne, 1987; 8 (10): 362-71.

[16] Clouet J., Simon H., Sellal O., Grimandi G., Duveau D. Le point sur les prothèses valvulaires. Phram Hosp, 2006; 41, 165 (15): 109-23. 